\title{
Distinctive electrophoretic isoenzyme profiles in Saccharomyces sensu stricto
}

\author{
Filomena L. Duarte, ${ }^{1,2}$ Célia Pais, ${ }^{2}$ Isabel Spencer-Martins ${ }^{3}$ \\ and Cecília Leão ${ }^{2}$
}

Author for correspondence: Célia Pais. Fax: +351 53678 980. e-mail: cpais@bio.uminho.pt

\footnotetext{
1 National Station for Viticulture and Enology, National Institute for Agrarian Research, 2560 Dois Portos, Portugal

2 Biology Department, University of Minho, Campus de Gualtar, 4709 Braga, Portugal

3 Biotechnology Unit, Faculty of Sciences and Technology, New University of Lisbon, 2825 Monte de Caparica, Portugal
}

\begin{abstract}
Genetic variation among $\mathbf{3 5}$ strains representing the four currently recognized species of Saccharomyces sensu stricto (Saccharomyces cerevisiae, Saccharomyces bayanus, Saccharomyces pastorianus/carlsbergensis and Saccharomyces paradoxus) was estimated by analysing the electrophoretic mobilities of nonspecific esterases, acid phosphatase, lactate dehydrogenase and glucose-6-phosphate dehydrogenase isoenzymes. Twenty-two electrophoretic types were identified, a result in agreement with the phenotypic and genetic polymorphisms reported for this group of yeasts. However, the four species were clearly distinguishable based on the patterns obtained using three of the enzymes assayed, the resolving power not being improved by the introduction of data correspondent to lactate dehydrogenase. The overall diversity was higher among $\mathbf{S}$. cerevisiae isolates, in contrast with S. paradoxus which showed only two patterns, one of which was common to four of the five strains studied. Concordant results from the application of the method and DNA hybridization experiments demonstrate its value for identification purposes.
\end{abstract}

Keywords: Saccharomyces, isoenzyme profiling, yeasts

\section{INTRODUCTION}

Common phenotypic criteria used in yeast identification often give unsatisfactory results. An illustrative example is provided by the group of species designated as Saccharomyces sensu stricto, which includes the most important strains in yeast based industries, such as baking, brewing and wine making. Most of the early controversy associated with a practical, though inconsistent, definition of species belonging to this group was centred on the ability to ferment and assimilate particular carbohydrates. The determination of the extent of DNA relatedness shed some light on the taxonomic relationships within the complex and revealed the four currently recognized species Saccharomyces cerevisiae, Saccharomyces bayanus, Saccharomyces pastorianus and Saccharomyces paradoxus (Vaughan Martini \& Martini, 1989).

Potentially useful typing methods have been applied to elucidate taxonomic and evolutionary relationships among species of this yeast group, including the study of serological properties (Campbell, 1972; Fukazawa

Abbreviation: ET, electrophoretic type. et al., 1980), cellular fatty acid analysis (Augustyn et $a l ., 1991$ ), electrophoretic karyotyping (Naumov et al., 1992; Vaughan Martini et al., 1993; Tornai-Lehoczki \& Dlauchy, 1996), restriction analysis of the mitochondrial DNA (Guillamón et al., 1994), sequencing of regions of the ribosomal nucleic acids (Kurtzman \& Robnett, 1991; Molina et al., 1992; James et al., 1997; Montrocher et al., 1998) and PCR-based methods for the detection of different genetic polymorphisms (de Barros Lopes et al., 1998). Moreover, the assessment of physiological and biochemical differential traits in Saccharomyces sensu stricto has been extended to nonconventional characteristics, such as the presence/ absence of active fructose transport and the ability to grow below/above $35^{\circ} \mathrm{C}$ which separate the pair $S$. bayanus $/ S$. pastorianus from $S$. cerevisiae/S. paradoxus (Rodrigues de Sousa et al., 1995). However, no user-friendly sensitive and reliable method was described that would allow the rapid identification of isolates while avoiding more sophisticated equipment and laborious procedures.

Electrophoretic enzyme polymorphism enables systematic and evolutionary genetic analysis and has been widely applied as a standard method both in the 
Trable 1. Strains studied and their sources of isolation

Abbreviations: IGC, Instituto Gulbenkian de Ciência, former location of Portuguese Yeast Culture Collection (PYCC), now at New University of Lisbon, Monte de Caparica, Portugal; CBS, Centraalbureau voor Schimmelcultures, Delft, Netherlands; DBVPG, Dipartimento di Biologia Vegetale, Università di Perugia, Italy. T, type strain; NT, neotype.

\begin{tabular}{|c|c|c|c|}
\hline \multirow[t]{2}{*}{ Species } & \multicolumn{2}{|r|}{ Strain } & \multirow[t]{2}{*}{ Isolation source } \\
\hline & IGC no. & Other collection nos & \\
\hline \multicolumn{4}{|c|}{ S. cerevisiae } \\
\hline & $4455^{\mathrm{NT}}$ & CBS 1171 & Beer (top yeast) \\
\hline & 2608 & CBS 1782 & Superattenuated beer \\
\hline & 2917 & CBS 429 & Fermenting grape juice \\
\hline & 3507 & & Human vaginitis \\
\hline & 3977 & CBS 1190 & \\
\hline & 4072 & & Commercial wine yeast \\
\hline & 4240 & & Wine, Portugal \\
\hline & 4543 & & Fermenting grape must, Austria \\
\hline & 4891 & & Olive brine \\
\hline & 5053 & & Fermenting grape must, Portugal \\
\hline & 5054 & & Fermenting grape must, Portugal \\
\hline & 5055 & & Fermenting grape must, Portugal \\
\hline & 5056 & & Fermenting grape must, Portugal \\
\hline & 5057 & & Fermenting grape must, Portugal \\
\hline & $5466^{*+}$ & & Commercial wine yeast \\
\hline & $5318^{*}$ & & Domestic bread leaven, Portugal \\
\hline & $5319^{*}$ & & Domestic bread leaven, Portugal \\
\hline & $5320^{*}$ & & Domestic bread leaven, Portugal \\
\hline & $5325^{*}$ & & Commercial baker's yeast \\
\hline & $5326^{*}$ & & Domestic bread leaven, Portugal \\
\hline \multicolumn{4}{|c|}{ S. pastorianus } \\
\hline & $4601^{\mathrm{NT}}$ & DBVPG 6047, CBS 1538 & Beer \\
\hline & 4261 & & Brewer's yeast, South Africa \\
\hline & 4457 & CBS 1513 & Beer (bottom yeast) \\
\hline & 4579 & CBS 1260 & Unknown \\
\hline & 4580 & CBS 1486 & Beer (bottom yeast) \\
\hline \multicolumn{4}{|c|}{ S. bayanus } \\
\hline & $4456^{\mathrm{T}}$ & CBS 380 & Turbid beer \\
\hline & 4565 & CBS 378 & Beer \\
\hline & 4567 & CBS 395 & Currant juice \\
\hline & 4568 & CBS 424 & Pear juice \\
\hline & 4569 & CBS 425 & Fermenting apple juice \\
\hline \multicolumn{4}{|c|}{ S. paradoxus } \\
\hline & $4570^{\mathrm{NT}}$ & CBS 432 & Tree exudate \\
\hline & 4576 & CBS 406 & Oak exudate \\
\hline & 4577 & CBS 2980 & Drosophila sp. \\
\hline & 4578 & CBS 5829 & Soil \\
\hline & 4656 & DBVPG 6489 & Tree exudate \\
\hline
\end{tabular}

* Strains identified only by conventional phenotypic methods (van der Walt \& Yarrow, 1984).

† Strain commercialized as $S$. bayanus.

taxonomy of prokaryotes and eukaryotes (Selander et al., 1986). In yeasts, a few studies evaluate the usefulness of this approach as a taxonomic tool (e.g. Baptist \& Kurtzman, 1976; Sidenberg \& Lachance, $1986)$ and to type clinical isolates of pathogenic species
(Brandt et al., 1993; Doebbeling et al., 1993; Lehmann et al., 1989). The Saccharomyces sensu stricto group also received some attention, but either because only reference strains were used or because the identification of the strains was not authenticated by whole- 
genome comparisons, the conclusions were not sound and a clear separation of species was not achieved (Yamazaki et al., 1983; Lewicka et al., 1995).

The purpose of the present work was to assess genetic variation among collection strains and new isolates belonging to species of the Saccharomyces sensu stricto group by the electrophoretic analysis of selected isoenzymes, and to further evaluate the usefulness of the technique for a rapid and sensitive identification of strains from this industrially important group of yeasts.

\section{METHODS}

Yeast strains and growth conditions. The yeast strains used in this study are listed in Table 1. Most of the strains tested were previously identified by DNA-DNA reassociation studies (Rodrigues de Sousa et al., 1995) and were obtained from the Portuguese Yeast Culture Collection (PYCC), New University of Lisbon, Portugal. Stock cultures were maintained on yeast extract-peptone-glucose-agar (YEPG) at $4{ }^{\circ} \mathrm{C}$. Yeasts were grown in a medium containing $0.5 \%(\mathrm{w} / \mathrm{v})$ Bacto-yeast extract, $1 \%(\mathrm{w} / \mathrm{v})$ Bacto-peptone and $2 \%(\mathrm{w} / \mathrm{v})$ glucose on a rotary shaker $(170$ r.p.m. $)$, at $25^{\circ} \mathrm{C}$. When grown up to late-exponential phase $\left(\mathrm{OD}_{640}=10-12\right)$, cells were harvested by centrifugation $\left(5000 \mathrm{~g}, 4^{\circ} \mathrm{C}\right.$ for $\left.7 \mathrm{~min}\right)$, washed twice with $20 \mathrm{ml}$ buffer I $(3.2 \mathrm{mM}$ Tris $/ \mathrm{HCl} \mathrm{pH} \mathrm{7)}$ and stored at $-20{ }^{\circ} \mathrm{C}$ until further utilization.

Preparation of extracts for electrophoresis. For protein extraction, cells were disrupted with $1 \mathrm{~g} 0.5 \mathrm{~mm}$ diameter glass beads and $1 \mathrm{ml}$ buffer II (60 mM Tris/ $\mathrm{HCl} \mathrm{pH} \mathrm{6.8)} \mathrm{by}$ vortexing $1 \mathrm{~min}$ and cooling the tube on ice for another minute. This operation was repeated at least eight times, until more than $70 \%$ of the cells were disrupted. Insoluble debris and undisrupted cells were removed by centrifugation at $15000 \mathrm{~g}, 0{ }^{\circ} \mathrm{C}$ for $30 \mathrm{~min}$. Protein extracts were stored at $-20^{\circ} \mathrm{C}$

Electrophoresis and detection of enzyme activity. Four enzymes, esterase (EST, EC 3.1.1.1), acid phosphatase (ACP, EC 3.1.3.2), glucose-6-phosphate dehydrogenase (G6PD, EC 1.1.1.49) and lactate dehydrogenase (LDH, EC 1.1.1.27), were assayed. Glycerol $(10 \% \mathrm{v} / \mathrm{v})$ and bromophenol blue $(0.001 \%, \mathrm{w} / \mathrm{v})$ were added to protein samples. Electrophoresis was run on a $0.75 \mathrm{~mm}$ thick, $7.5 \%$ (w/v) (for ACP, G6PD and LDH and $8 \%$, w/v, for EST) non-denaturating polyacrylamide gel, overlaid with $3.75 \%$ w/v polyacrylamide stacking gel (Hames, 1981), using the Mighty Small II apparatus from Hoefer/Pharmacia. Electrophoresis was carried out at $5{ }^{\circ} \mathrm{C}$ with a constant initial voltage of $100 \mathrm{~V}$ for $30 \mathrm{~min}$, followed by $150 \mathrm{~V}$ until the tracking dye (bromophenol blue) reached the bottom of the gel (approx. 1.5 h). Staining procedures were similar to those previously described by Pais (1990), for EST and G6PD, by Eiras-Dias (1994) for ACP, and by Shaw \& Prasad (1970) for LDH.

Analysis of data. The relative electrophoretic mobilities $\left(R_{M}\right)$ of the enzyme bands were calculated as the ratio of the migration of each band to that of the tracking dye and adjusted by comparison with the values obtained for $S$. cerevisiae IGC 4072 (IGC, Instituto Gulbenkian de Ciência, the former location of the Portuguese Yeast Culture Collection), electrophoresed on the same gel and used as a reference. Numerical analysis was performed with NTSYS-pc software package (Rohlf, 1992). For each enzyme, original matrices consisted of presence (1) or absence ( 0 ) of a band with a given $R_{M}$ for every studied strain. Relationships between electrophoretic types of strains were calculated using the Dice similarity coefficient. Dendrograms were generated by applying unweighted pair group average linkage clustering (UPGMA) to the similarity matrix obtained (Sneath \& Sokal, 1973). In order to test the fit of the clustering, the co-phenetic correlation coefficient was calculated.

Reproducibility of the results. The experiments were repeated at least three times with protein extracts prepared from different cultures of the same strain, and the results obtained were reproducible.

\section{RESULTS AND DISCUSSION}

\section{Isoenzyme analysis}

A total of 35 strains, belonging to $S$. cerevisiae, $S$. bayanus, $S$. pastorianus and $S$. paradoxus were analysed for their electrophoretic profiles of EST, ACP, G6PD and LDH. Twenty-two distinct electrophoretic types (ETs) were obtained for the four enzyme systems investigated (Fig. 1), a result in agreement with the recognized heterogeneity within the Saccharomyces sensu stricto complex. Representative phenotypes are shown in Fig. 2.

All strains of $S$. cerevisiae had a single band pattern for EST and G6PD, except strain IGC 2917 which, in both cases, presented two additional slow migrating bands. These patterns were unique for this species. Noteworthy, this is the type strain of Saccharomyces oviformis, which was also reported to behave differently from the other $S$. cerevisiae strains with respect to the maximum temperature for growth (Rodrigues de Sousa et al., 1995). The same authors suggested that it might be a hybrid of $S$. cerevisiae and S. bayanus, bearing a higher $(87 \%)$ nuclear DNA relatedness with the former species (Vaughan Martini \& Martini, 1987). Our results do not add up to this hypothesis, since it also displays an ET distinct from those observed in S. bayanus (Fig. 1). For ACP and LDH, S. cerevisiae showed a higher polymorphism, with four and eight different patterns, respectively. However, with respect to ACP, all strains except the type strain displayed the same band, not found in the other species of the group. The results indicate that LDH isoenzymes account for most of the polymorphism observed. In fact, the $20 \mathrm{~S}$. cerevisiae strains studied could be grouped in 11 different ETs, this number being reduced to only five when LDH was not considered (Fig. 1). In similar studies, based on a higher number of enzymes, the authors report as many different ETs as the number of strains under study (Subden et al., 1982; Yamazaki et al., 1983; Poncet et al., 1992). In contrast, Lewicka et al. (1995) in studies of genetic diversity based on multilocus starch electrophoresis of seven isoenzymes described 11 different ETs for 27 strains of $S$. cerevisiae, which is in accordance with our results and indicates that the discriminatory power of the enzymes varies significantly. It looks as if it is not the number of enzymes tested that matters but to find the appropriate set with 


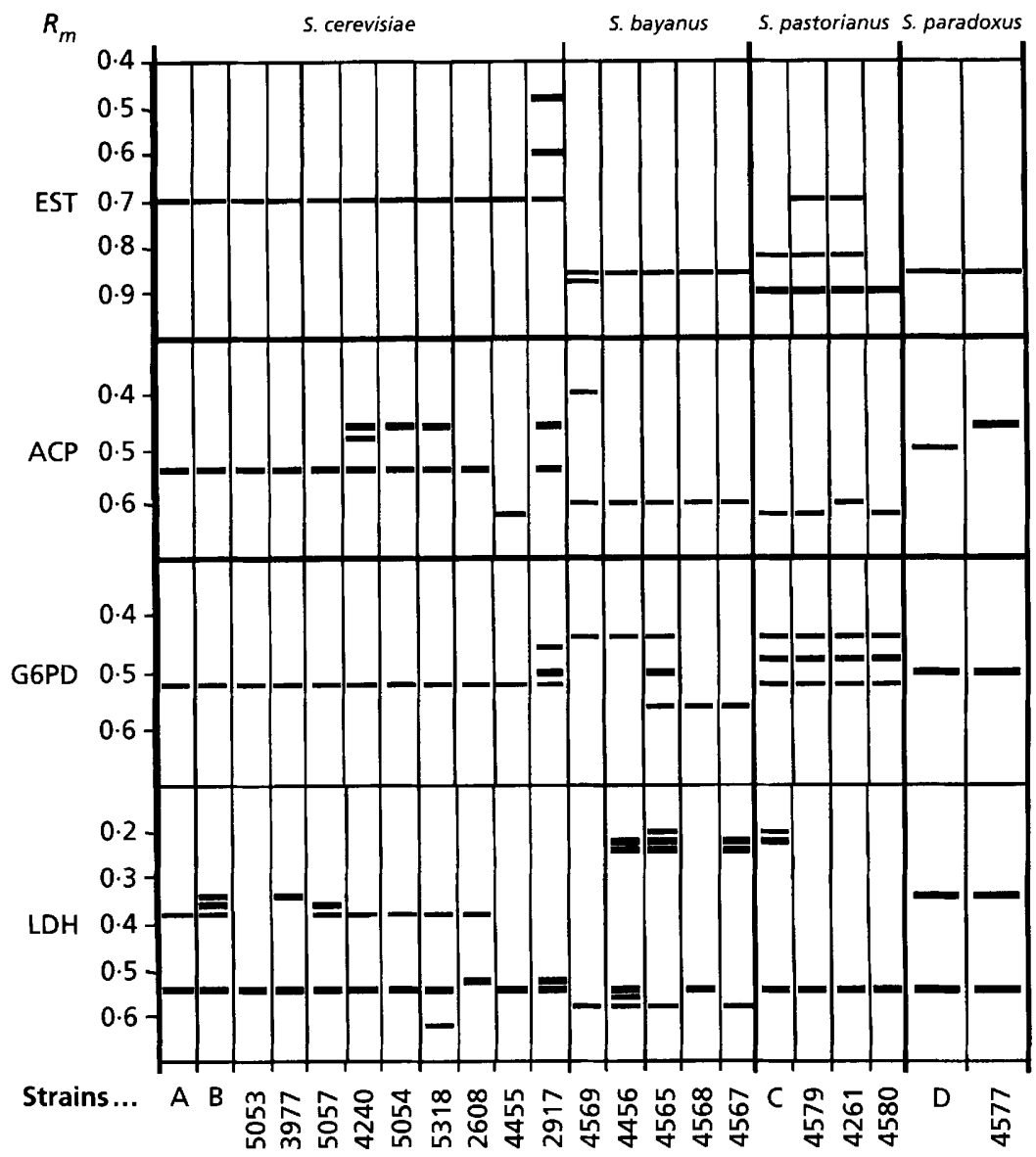

Fig. 1. Schematic representation of patterns of enzymic activity (EST, ACP, G6PD and LDH) found in the 35 strains belonging to species of Saccharomyces sensu stricto after PAGE. $A=3507,4072,4543,4891,5055$, $5056,5320,5466 ; B=5319,5325,5326 ; C=$ 4457,$4601 ; D=4570,4576,4578,4656$. the highest resolving power at the specific level required.

Regarding the isoenzymes studied, $S$. bayanus presented some heterogeneity, the five strains displaying five different ETs. The strains presented a single band for EST and ACP isoenzymes, except for one strain (IGC 4569) which showed a fast-migrating band in the case of EST, and a much slower one in the case of ACP. Higher polymorphisms were found for G6PD and LDH with three and five patterns, respectively. Interestingly, several authors who used other molecular typing methods, such as electrophoretic karyotyping, refer to $S$. bayanus as a very homogeneous species (Naumov et al., 1992; TornaiLehoczki \& Dlauchy, 1996).

In what concerns $S$. pastorianus, EST activity was the most polymorphic, three different patterns being observed. No intraspecific variability among the five strains studied was detected in G6PD phenotypes, whereas for ACP only one strain presented a different pattern. For LDH two distinct patterns were found, but with one main band in common.

$S$. paradoxus appeared to be the most homogeneous species of the group, since all the strains studied presented a single pattern for EST, LDH and G6PD. However, only the G6PD pattern was unique and characteristic of this species. This intraspecific hom- ogeneity may be related to the fact that strains of this species can only be found in natural habitats. A recent report on the differentiation of European and Asian populations of $S$. paradoxus, by allozyme electrophoresis, revealed a significant genetic diversity among isolates of the two geographically separate regions, but each group was in itself very homogeneous (Naumov et al., 1997).

Globally, we found that EST patterns enabled a clear distinction between $S$. cerevisiae and the other species, because most strains showed a single, common band, not found in the other three species. However, esterase patterns alone did not allow the separation of the other three species since strains of $S$. bayanus and $S$. paradoxus displayed similar EST patterns. When ACP phenotypes were considered, despite some intraspecific variability, the discrimination between $S$. bayanus and $S$. paradoxus could be improved. To quantify the resolving capacity of the electrophoretic phenotypes of all isoenzymes, which is difficult to achieve only by visual examination, the data were subjected to numerical analysis.

\section{Numerical analysis of isoenzyme profiles}

To further evaluate the relatedness among the species under study and the value of the method used for taxonomic purposes, numerical analysis, namely clus- 


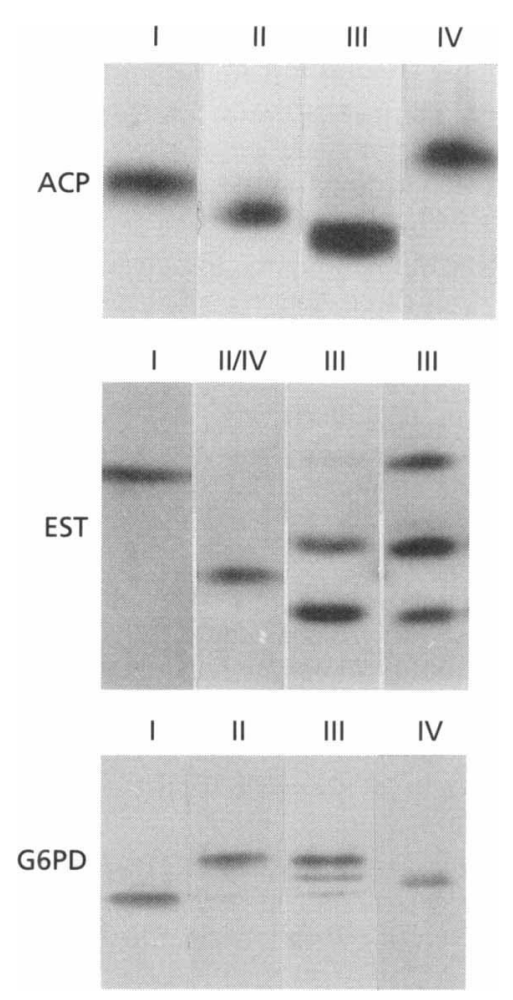

Fig. 2. Representative electrophoretic variants of EST, ACP and G6PD found after polyacrylamide gel electrophoresis in cell extracts of the Saccharomyces strains studied. I = S. cerevisiae; II $=S$. bayanus; $I I I=S$. pastorianus; $I V=S$. paradoxus.

ter analysis, was applied to the data obtained. Four meaningless clusters, in terms of strain conspecificity, were obtained from isoenzyme variation ( 31 band positions) of the four enzyme systems tested (data not shown). However, the similarity within some clusters was almost as high as among clusters, indicating low consistency of the grouping. As mentioned before, LDH was the most polymorphic enzyme, accounting for a great increase of the intraspecific variation. The consistency of the clusters could, therefore, be improved if LDH data were not included. The dendrogram thus obtained is shown in Fig. 3. The level of similarity observed within each cluster was much higher $(>0 \cdot 50)$ than among clusters $(>0 \cdot 30)$, and a clear separation between the four Saccharomyces sensu stricto species was achieved. The calculated cophenetic correlation coefficient $(0.986)$ indicated that the fit for the cluster analysis was very high.

A striking aspect when analysing these results is the homogeneity found among $S$. cerevisae strains, which are all clustered in one group despite their different isolation sources. Two separate branches of this cluster are constituted by the aforementioned $S$. oviformis strain and by the type strain of $S$. cerevisiae, the latter due to its exceptional behaviour with respect to ACP. Closely related to this group are the strains of $S$. pastorianus. In respect to $S$. bayanus and $S$. paradoxus, they are included in two distinct but closely related

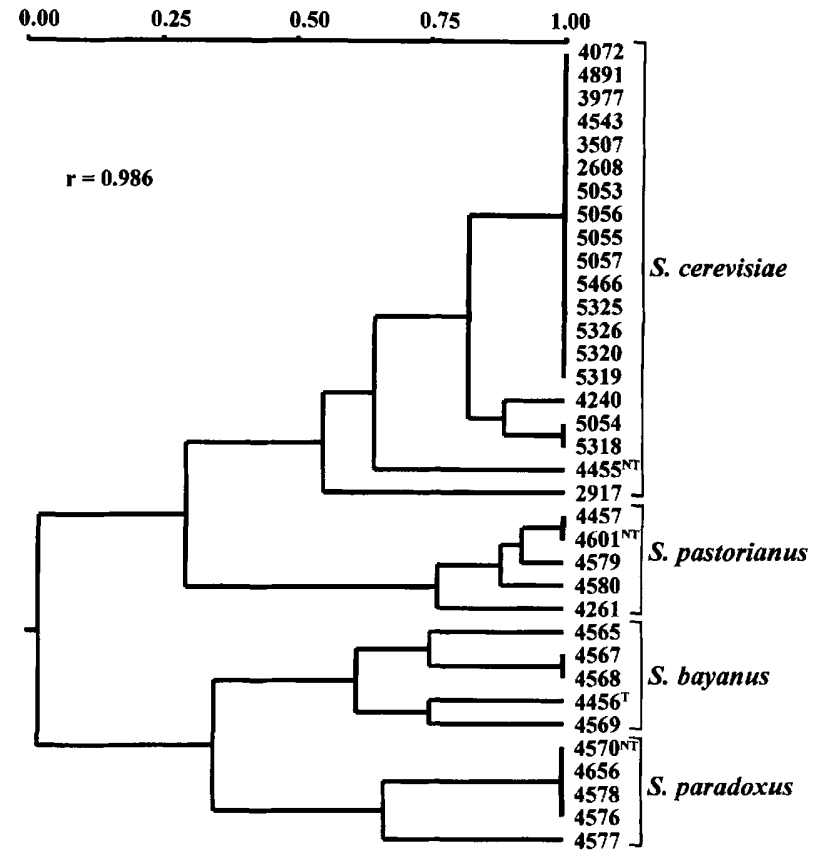

Fig. 3. Dendrogram showing the degree of similarity of electrophoretic types among strains of the Saccharomyces sensu stricto complex based on the data obtained from the EST, ACP and G6PD isoenzyme patterns. Scale at top represents a numerical measure of similarity. $r$, co-phenetic correlation coefficient.

clusters. The higher similarity found between $S$. cerevisiae and $S$. pastorianus is in accordance with rDNA restriction pattern analysis (Molina et al., 1992), though these authors did not include the type strains of the corresponding species in their study, and also with the higher percentage of DNA-DNA reassociation found between both species by Rodrigues de Sousa et al. (1995). On the contrary, most of the works on the characterization of this group of yeasts, namely electrophoretic karyotyping (Naumov et al., 1992), mtDNA restriction patterns (Guillamón et al., 1994) and ribosomal nucleic acids sequence data (Kurtzman \& Robnett, 1991; James et al., 1997; Montrocher et al., 1998) found a closer relationship between $S$. cerevisiae and $S$. paradoxus. These discrepancies, though not solved by the present data, do not lead to any ambiguous interpretation of our results, which provide an interspecific differentiation of isoenzyme patterns.

Our results differ from those obtained by other authors in studies on the electrophoretic mobility of isoenzymes in the species examined of the genus Saccharomyces as they generally present a greater intraspecific diversity in patterns and fail to separate the four species belonging to Saccharomyces sensu stricto (Subden et al., 1982; Yamazaki et al., 1983; Poncet et al., 1992; Lewicka et al., 1995). The experimental conditions, namely the use of certified strains and the time of harvesting the cells, may account for the 
consistency of these results. We have verified (data not shown) that at the stationary phase of growth EST patterns present additional fainter bands that probably lead to higher polymorphism but to less intraspecific discrimination. It should be again emphasized that the choice of enzymes appear also to be of major importance.

It should be pointed out that other eventual yeast contaminants in fermentation-related environments, such as Torulaspora delbrueckii, Zygosaccharomyces bailii and Pichia membranifaciens, have also been examined using the same methodology and yielded distinct electrophoretic types (results not shown).

In view of the present results we consider that the electrophoretic profiles of EST, ACP and G6PD isoenzymes are good markers for species differentiation in Saccharomyces sensu stricto and a potentially useful tool for the rapid identification of isolates in this group of industrially important yeasts. In practice, every new isolate belonging to the group can be analysed, following the procedure described for determination of isoenzyme patterns. The results can then be computer-compared, in a user-friendly way, to the available data on the strains of Saccharomyces sensu stricto studied and thereby be automatically assigned to one of the four species.

\section{ACKNOWLEDGEMENTS}

The authors are grateful for the technical assistance of M. Filomena Alemāo. This work was supported by the EU Project AIR-CT93-0830.

\section{REFERENCES}

Augustyn, O. P. H., Ferreira, D. \& Kock, J. L. F. (1991). Differentiation between yeast species, and strains within a species, by cellular fatty acid analysis. 4. Saccharomyces sensu stricto, Hanseniaspora, Saccharomycodes and Wickerhamiella. Syst Appl Microbiol 14, 324-334.

Baptist, J. N. \& Kurtzman, C. (1976). Comparative enzyme patterns in Cryptococcus laurentii and its taxonomic varieties. Mycologia 68, 1195-1203.

de Barros Lopes, M., Soden, A., Martens, A. L., Henschke, P. A. \& Langridge, P. (1998). Differentiation and species identification of yeasts using PCR. Int J Syst Bacteriol 48, 279-286.

Brandt, M. E., Bragg, S. L. \& Pinner, R. W. (1993). Multilocus enzyme typing of Cryptococcus neoformans. J Clin Microbiol 31, 2819-2823.

Campbell, I. (1972). Numerical analysis of the genera Saccharomyces and Kluyveromyces. J Gen Microbiol 73, 279-301.

Doebbeling, B. N., Lehmann, P. F., Hollis, R. J., Wu, L.-C., Widmer, A. F., Voss, A. \& Pfaller, M. A. (1993). Comparision of pulsedfield gel electrophoresis with isoenzyme profiles as a typing system for Candida tropicalis. Clin Infect Dis 16, 377-383.

Eiras-Dias, J. E. (1994). Isoenzyme polymorphism in the identification of Vitis vinifera L. cultivars. PhD thesis, Instituto Superior de Agronomia, Technical University of Lisbon, Portugal (in Portuguese).

Fukazawa, Y., Shinoda, T., Nishikawa, A. \& Nakase, T. (1980). Synonymy of Saccharomyces cerevisiae Hansen 1883 and
Saccharomyces uvarum Beijerinck 1898: significance of cell wall antigenes in yeast classification. Int J Syst Bacteriol 30, 196-205.

Guillamón, J. M., Barrio, E., Huerta, T. \& Querol, A. (1994). Rapid characterization of four species of the Saccharomyces sensu stricto complex according to mitochondrial DNA patterns. Int J Syst Bacteriol 44, 708-714.

Hames, B. D. (1981). Introduction to polyacrylamide gel electrophoresis. In Gel Electrophoresis of Proteins - a Practical Approach, pp. 1-91. Edited by B. D. Hames \& D. Rickwood. Oxford: IRL Press.

James, S. A., Cai, J., Roberts, I. N. \& Collins, M. D. (1997). A phylogenetic analysis of the genus Saccharomyces based on 18S rRNA gene sequences: description of Saccharomyces kunashirensis sp. nov. and Saccharomyces martiniae sp. nov. Int $J$ Syst Bacteriol 47, 453-460.

Kurtzman, C. \& Robnett, C. J. (1991). Phylogenetic relationship among species of Saccharomyces, Schizosaccharomyces, Debaryomyces and Schwanniomyces determined from partial ribosomal RNA sequences. Yeast 7, 61-72.

Lehmann, P. F., Kemker, B. J., Hsiao, C.-B. \& Dev, S. (1989). Isoenzyme biotypes of Candida species. J Clin Microbiol 27, 2514-2521.

Lewicka, K., Mallie, M. \& Bastide, J.-M. (1995). Genetic variability in the Saccharomyces sensu stricto complex revealed by multilocus enzyme electrophoresis. Int $J$ Syst Bacteriol 45, 538-543.

Molina, F. I., Inoue, T. \& Jong, S.-C. (1992). Ribosomal DNA restriction analysis reveals genetic heterogeneity in Saccharomyces cerevisiae Meyen ex Hansen. Int J Syst Bacteriol 42, 499-502.

Montrocher, R., Verner, M.-C., Briolay, J., Gautier, C. \& Marmeisse, R. (1998). Phylogenetic analysis of the Saccharomyces cerevisiae group based on polymorphisms of rDNA spacer sequences. Int $J$ Syst Bacteriol 48, 295-303.

Naumov, G. I., Naumova, E. S., Lantto, R. A., Louis, E. J. \& Korhola, M. (1992). Genetic homology between Saccharomyces cerevisiae and its sibling species $S$. paradoxus and $S$. bayanus: electrophoretic karyotypes. Yeast 8, 599-612.

Naumov, G. I., Naumova, E. S. \& Sniegowski, P. D. (1997). Differentiation of European and Far East Asian populations of Saccharomyces paradoxus by allozyme analysis. Int $J$ Syst Bacteriol 47, 341-344.

Pais, C. (1990). Biochemical studies applied to the taxonomy and physiology of plant parasitic nematodes. $\mathrm{PhD}$ thesis, Universidade do Minho, Braga.

Poncet, S., Montrocher, R. \& Couble, A. (1992). Electrophoretic isoenzyme patterns of some yeast strains used in wine industry. Cryptogam Mycol 13, 283-293.

Rodrigues de Sousa, H., Madeira-Lopes, A. \& Spencer-Martins, I. (1995). The significance of active fructose transport and maximum temperature for growth in the taxonomy of Saccharomyces sensu stricto. Syst Appl Microbiol 18, 44-51.

Rohlf, J. F. (1992). NTSYS-pc Numerical Taxonomy and Multivariate System version 1.70. New York: Applied Biostatistics.

Selander, R. K., Caugant, D. A., Ochman, H., Musser, J. M., Gilmour, M. N. \& Whittman, T. S. (1986). Methods of multilocus enzyme electrophoresis for bacterial population genetics and systematics. Appl Environ Microbiol 51, 873-884.

Shaw, C. \& Prasad, R. (1970). Starch gel electrophoresis of enzymes - a compilation of recipes. Biochem Genet 4, 297-320.

Sidenberg, D. G. \& Lachance, M.-A. (1986). Electrophoretic isoenzyme variation in Kluyveromyces populations and revision 
of Kluyveromyces marxianus (Hansen) van der Walt. Int J Syst Bacteriol 36, 94-102.

Sneath, P. H. A. \& Sokal, R. R. (1973). Numerical Taxonomy. The Principles and Practice of Numerical Classification. San Francisco: Freeman.

Subden, R. E., Irwin, D., Cunningham, J. D. \& Meiering, A. G. (1982). Wine yeast isozymes. I. Genetic differences in 18 stock cultures. Can J Microbiol 28, 1047-1050.

Tornai-Lehoczki, J. \& Dlauchy, D. (1996). An opportunity to distinguish species of Saccharomyces sensu stricto by electrophoretic separation of the larger chromosomes. Lett Appl Microbiol 23, 227-230.

Vaughan Martini, A. \& Martini, A. (1987). Three newly delimited species of Saccharomyces sensu stricto. Antonie Leeuwenhoek 53, 77-84.
Vaughan Martini, A. \& Martini, A. (1989). A proposal for correct nomenclature of the domesticated species of the genus Saccharomyces. In Biotechnology Applications in Beverage Production, pp. 1-16. Edited by C. Cantarelli \& G. Lanzarini. London: Elsevier.

Vaughan Martini, A., Martini, A. \& Cardinali, G. (1993). Electrophoretic karyotyping as a taxonomic tool in the genus Saccharomyces. Antonie Leeuwenhoek 63, 145-146.

van der Walt, J. P. \& Yarrow, D. (1984). Methods for isolation, maintenance, classification and identification of yeasts. In The Yeasts, a Taxonomic Study, pp. 45-104. Edited by N. J. W. Kreger van Rij. Amsterdam: Elsevier.

Yamazaki, M., Goto, S. \& Komagata, K. (1983). An electrophoretic comparison of the enzymes of Saccharomyces yeasts. J Gen Appl Microbiol 29, 305-318. 